\title{
Lesson of the month 1: Spontaneous septic thrombophlebitis presenting with bacteraemia diagnosed by PET-CT scan
}

\author{
Authors: Patrick Harnett ${ }^{A}$ and Shaifali Jain ${ }^{B}$
}

Spontaneous septic thrombophlebitis is a rare complication of Staphylococcus aureus bacteraemia. Its true incidence is unknown as septic thrombus is not often considered as a source in the typical 'screen' of tests used to find the source of a bacteraemia. Positron emission tomography computerised tomography (PET-CT) is becoming increasingly available to physicians, is highly sensitive and yields specific anatomical information regarding abnormal metabolically active sites in infection, inflammation and neoplasia. In this case, PET-CT enabled the identification of the source of a septic thrombus and enabled focused management. PET-CT should be considered as part of the raft of tests used to identify an obscure source of fever/bacteraemia.

KEYWORDS: PET-CT, septic thrombophlebitis, Staphylococcus aureus

\section{Case history}

A 58-year-old Caucasian man presented to his primary care doctor following a 5-day febrile illness with lower back and leg pain. There was no weight loss or recent foreign travel. The patient had experienced episodes of uncontrolled intermittent shivering or rigors. He was referred to the emergency medical team for further evaluation.

Vital signs, including pulse, were normal on admission. Subsequent observations demonstrated an intermittent fever, which peaked at $38.2^{\circ} \mathrm{C}$ and was accompanied by a pulse of $110 \mathrm{bpm}$.

Physical examination showed normal heart sounds, normal abdominal findings and no sign of peripheral oedema. Breath sounds and oxygen saturations were normal. There were no splinter haemorrhages. Urine was positive for protein (trace) and negative for blood cells.

Preliminary blood tests showed an elevated white cell count $15.3 \times 10^{9} / \mathrm{L}$ and a C-reactive protein $(\mathrm{CRP})$ of $421 \mathrm{mg} / \mathrm{L}($ normal $<5)$.

The patient was admitted and treated with intravenous amoxicillin and clavulanic acid. Blood cultures taken at presentation yielded a strong growth of Staphylococcus aureus on the second day of admission. The isolates were sensitive to flucloxacillin.

Authors: A consultant general physician and nephrologist, Southend University Hospital NHS Foundation Trust, Southend, UK; ${ }^{B}$ consultant radiologist, Southend University Hospital NHS Foundation Trust, Southend, UK
Differential diagnoses considered included endocarditis, bacterial discitis, psoas abscess and other occult staphylococcal abscess. The patient underwent a number of preliminary investigations: a chest X-ray, computerised tomography (CT) urinary tract, magnetic resonance imaging (MRI) spine and transoesophageal echo, which were all within normal limits.

MRI spine demonstrated degenerative changes. Appearances were not consistent with a diagnosis of discitis. A transthoracic echocardiogram was normal.

Antibiotics were changed to intravenous flucloxacillin. A reduction in CRP to normal and resolution of fever occurred over the next 5 days.

A whole-body fluorodeoxyglucose (FDG) positron emission tomography (PET) CT scan was obtained.

Abnormal uptake was demonstrated in an area corresponding to the femoral vein with no other areas of abnormal avidity (Fig 1).

Ultrasound scanning of the vein demonstrated occlusive thrombus. No distal swelling of the limb occurred.

Repeat examination revealed no portal of entry, no signs of IV drug use. Review of the history and further examination did not yield any evidence of inoculation event but did reveal that varicose vein surgery had been conducted some 20 years previously. Review of the CT scan demonstrated the presence of metallic surgical clips for varicose veins. No evidence of track, sinus or abscess was evident in the usual sites employed for intravenous drug use.

\section{Discussion}

$S$ aureus is a ubiquitous human commensal and also common as a virulent human pathogen. Bacteraemia with $S$ aureus $(\mathrm{SAB})$ confers a mortality rate that ranges from $15-50 \%$. The incidence of SAB has been increasing over the last few decades. Much of this is accounted for by a rise in resistant infections, such as methicillin-resistant $S$ aureus (MRSA). Morbidity from SAB results from metastatic sites of infection, such as endocarditis and vertebral discitis. The site of infection remains obscure in approximately $25 \%$ of cases. ${ }^{1}$

Risk factors for SAB are well recognised and are commonly associated with an identifiable portal of entry, such intravenous drug use, medical invasive devices - including dialysis catheters, central venous lines and peripheral cannulas. Other risk factors include immunosuppression from viral infections or medications.

Community-acquired or spontaneous SAB in the absence of usual risk factors is unusual and mandates extensive investigation to establish cause and source. 


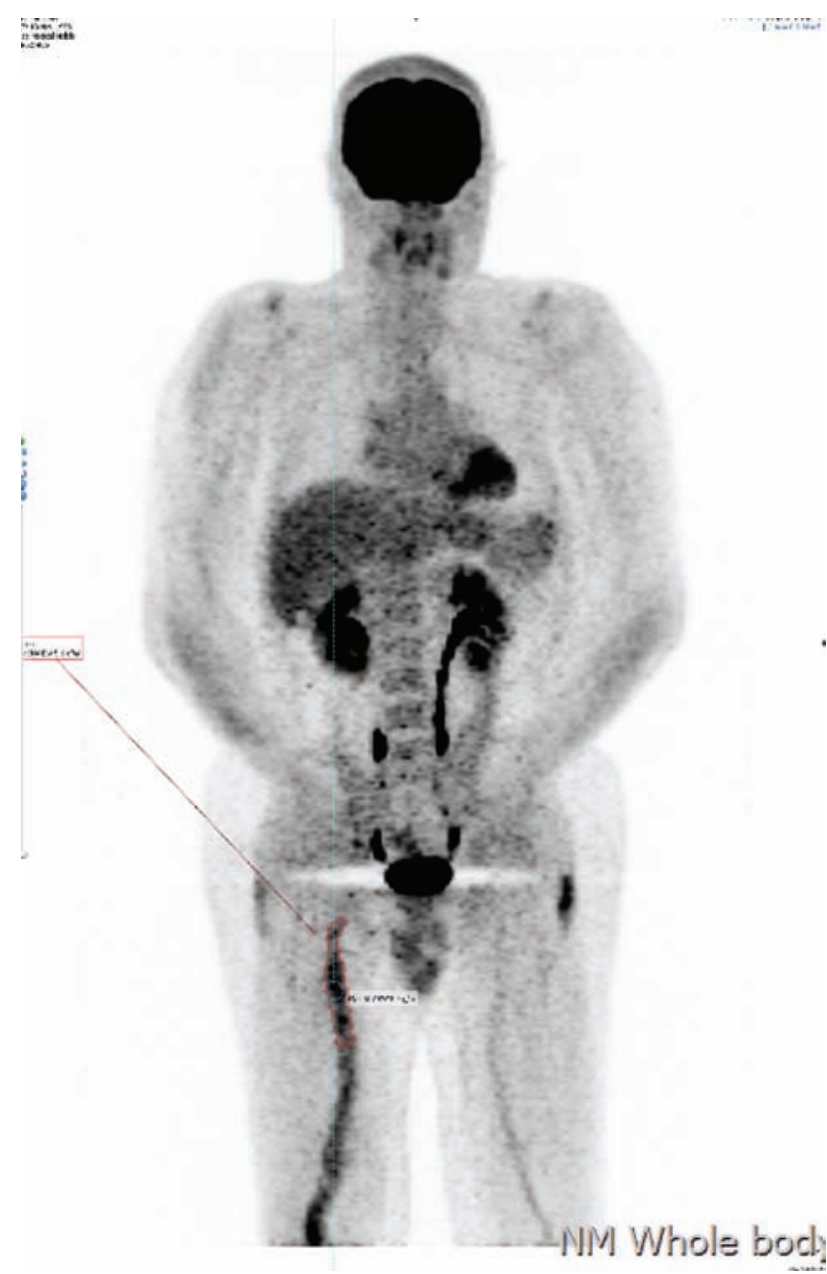

Fig 1. FDG PET-CT scan showing activity in the right femoral vein. $\mathrm{FDG}=$ fluorodeoxyglucose; $\mathrm{PET}-\mathrm{CT}$ = positron emission tomography computerised tomography

Two key principles of management of SAB have been proposed. ${ }^{2}$ Primarily, to identify the focus or source of bacteraemia and remove it and subsequently to administer a sufficiently long course of appropriate antibiotics in the case of a source that cannot be removed.

In our case the conventional approach of source identification involving hospital admission and extensive investigation and imaging did not reveal the source. The use of PET vividly demonstrated an abnormal metabolically active area corresponding to an infected deep-vein thrombus. PET-CT is a functional imaging technique that preferentially demonstrates metabolically active regions in the body. PET-CT involves the injection of FDG, a glucose analogue, with a positron emitting source, which is consumed by glucose avid processes such as brain activity, neoplastic growth or inflammation.

PET has become a widely used and well established technique for evaluation of patients with oncological disease. There is also emerging evidence for the use of PET in patients with pyrexia of unknown origin (PUO) or inflammation of unknown origin (IUO). The strength of PET in the context of PUO/IUO resides with the sensitivity and the negative predictive value. ${ }^{3}$
Septic or suppurative thrombophlebitis is bacterial or fungal infection of a thrombus in the great veins. In some reports the thrombus as a source of bacteraemia is thought to be as high as $8 \%$ of patients with positive blood cultures. ${ }^{4}$ It is well described in intravenous drug users. There is a common association with invasive devices, such as venous lines or dialysis catheters. It is recognised in bacterial infections of the pharynx causing septic thrombus in the jugular vein (Lemmiers syndrome). Septic thrombosis is known to occur as a complication of surgery in the pelvic veins.

Suppurative thrombophlebitis in proximal leg veins was found in $11 \%$ of intravenous drug users with infectious symptoms. ${ }^{5}$ Complications, including endocartidits septic arthritis and skin abscess, were common. The appearance of spontaneous suppurative thrombophlebitis is rare.

This case suggests a number of learning points:

$>$ Where the source of bacteraemia is obscure, FDG PET may be useful in localising the origin. The incidence of spontaneous septic thrombophlebitis is unknown. The proportion of SAB of unknown source represented by septic thrombophlebitis is unknown but may become clearer with the greater availability of FDG PET.

$>$ Identification of an infective venous thrombus alters subsequent management as anticoagulation, thrombophilia studies and vein imaging may be required.

$>$ A positive identification of the anatomical site negates the need for further imaging and permits shorter stay in hospital for investigation.

We found no published cases of septic thrombus related to surgical clips.

\section{Conflicts of interest}

The authors have no conflicts of interest to declare.

\section{Acknowlegements}

Consent was obtained from the patient for publication of the clinical details and image in this article.

\section{References}

1 Tong SY, Davis JS, Eichenberger E, Holland TL, Fowler VG Jr. Staphylococcus aureus infections: epidemiology, pathophysiology, clinical manifestations, and management. Clin Microbiol Rev 2015;28:603-61.

2 Thwaites GE, Edgeworth JD, Gkrania-Klotsas E et al. Clinical management of Staphylococcus aureus bacteraemia. Lancet Infect Dis 2011;11:208-22.

3 Balink H, Verberne HJ, Bennink RJ, van Eck-Smit BL. A rationale for the use of F18-FDG PET/CT in fever and inflammation of unknown origin. Int J Mol Imaging 2012;2012:165080.

4 Fowler VG Jr, Olsen MK, Corey GR et al. Clinical identifiers of complicated Staphylococcus aureus bacteremia. Arch Intern Med 2003;163:2066-72.

5 Mertz D, Khanlari B, Viktorin N, Battegay M, Fluckiger U. Less than 28 days of intravenous antibiotic treatment is sufficient for suppurative thrombophlebitis in injection drug users. Clin Infect Dis 2008;46:741-4.

Address for correspondence: Dr Patrick Harnett, Southend Hospital, Prittlewell Chase, Westcliff on Sea SSO ORY, UK. Email: patrick.harnett@southend.nhs.uk 